\title{
A Platform for Accurate Mass and Time (AMT) Analyses of Mass Spectrometry Data.
}

Authors: Damon H. May, Matt Fitzgibbon, Yan Liu, Ted Holzman, Jimmy Eng, C.J. Kemp, Jeff Whiteaker, Amanda Paulovich, Martin McIntosh*

\section{SUPPORTING INFORMATION}

\section{CPAS Integration}

The msInspect platform contains components for integration with the Computational Proteomics Analsysis System (CPAS). Specifically, msInspect may be used to browse a CPAS site and select a series of LC-MS/MS data files for use in building the AMT database. This client application uses the caBIG client API provided by CPAS, which conforms to caBIG silver level requirements.

Interaction with CPAS through this application is subject to the same authentication procedures used when logging into the CPAS website. After authentication through the msInspect client, the user can only see publicly-available folders and folders visible to that user. Figure 1 illustrates this interface.

The user can navigate through the hierarchy of available Folders and Experiments to find the files related to a database search of MS/MS data. This includes the input files (FASTA files, XML files 
containing search engine setings, and mzXML files containing spectra), output files (ProteinProphet files containing protein-level search results), and other files (Peptide-level search results). Any of these files may be downloaded to the user's computer.

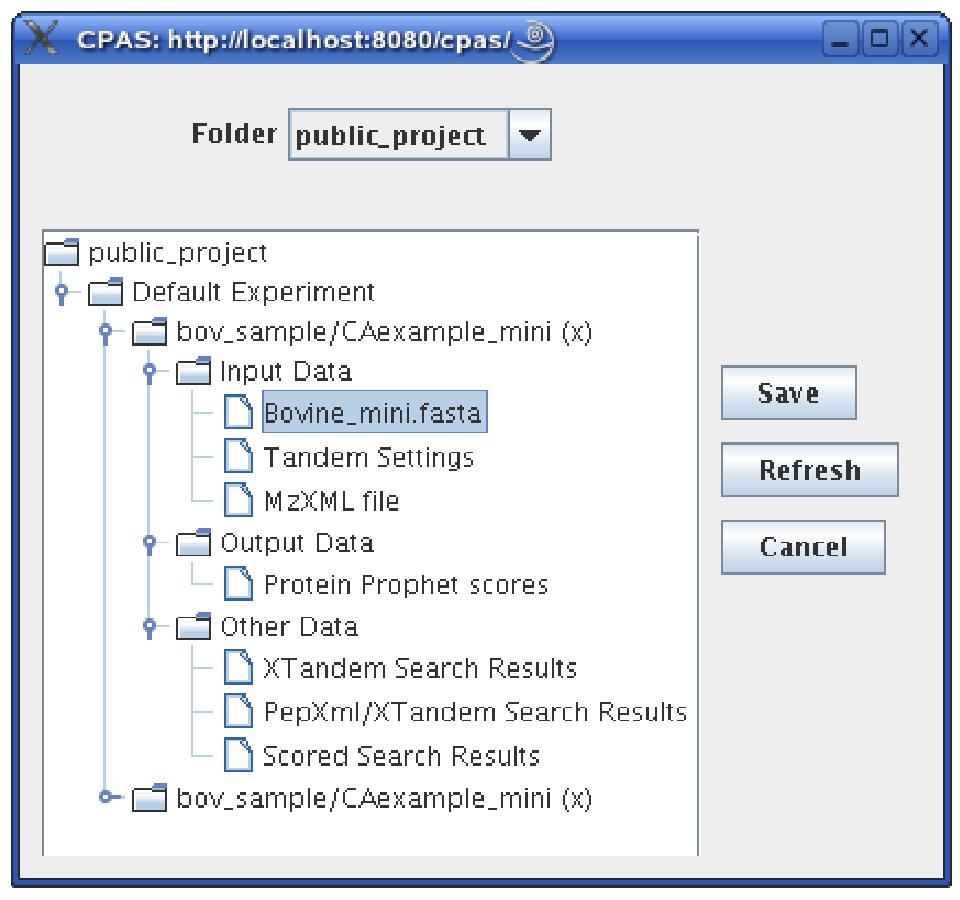

Figure 1. CPAS integration user interface.

\section{Robust Regression for Relating Retention Time to Hydrophobicity}

In order to assign normalized retention time values to LC-MS peptide observations, we need to determine the linear relationship between retention time and hydrophobicity for a particular experiment.

We begin with a set of LC-MS/MS peptide observations, from the same LC-MS run. We throw out all but the most confident peptides (PeptideProphet probably $>=0.95$ ). For each of these peptide observations, we use a hydrophobicity prediction algorithm to assign a Calculated Hydrophobicity value. This value is correlated with retention time - how tightly depends on the specific algorithm 
used. Next, we normalize that value to the same scale as the Normalized Retention Time values stored in our AMT database. Now, we have both a Normalized Calculated Hydrophobicity value and a Retention time value for each data point. We want to discover the linear relationship between those two quantities.

The linear model is estimated using robust regression techniques based on automated outlier detection approaches in order to be robust with respect to peptide identification errors.

First, we calculate the leverage of each peptide based on its retention time. Using only the peptide identifications with leverage $<=4 / \mathrm{n}$, we perform a linear regression to map from time to $\mathrm{H}$ and calculate the $\mathrm{H}$ value of all the identifications based on this mapping (Figure 2A).

Next, we calculate each peptide's studentized residual, based on its retention time and the $\mathrm{H}$ value assigned in the previous step. Then we perform a second regression, this time excluding any peptides with a studentized residual $>2$ (Figure $2 \mathrm{~B}$ ).

The $\mathrm{H}$ value for all peptides with studentized residual $<=2$ is then recalculated using this new mapping. We refer to the fitted value $\hat{H}$ as the "normalized retention time" for that peptide, and it is this value that is stored in the AMT database as representative of this peptide observation. 


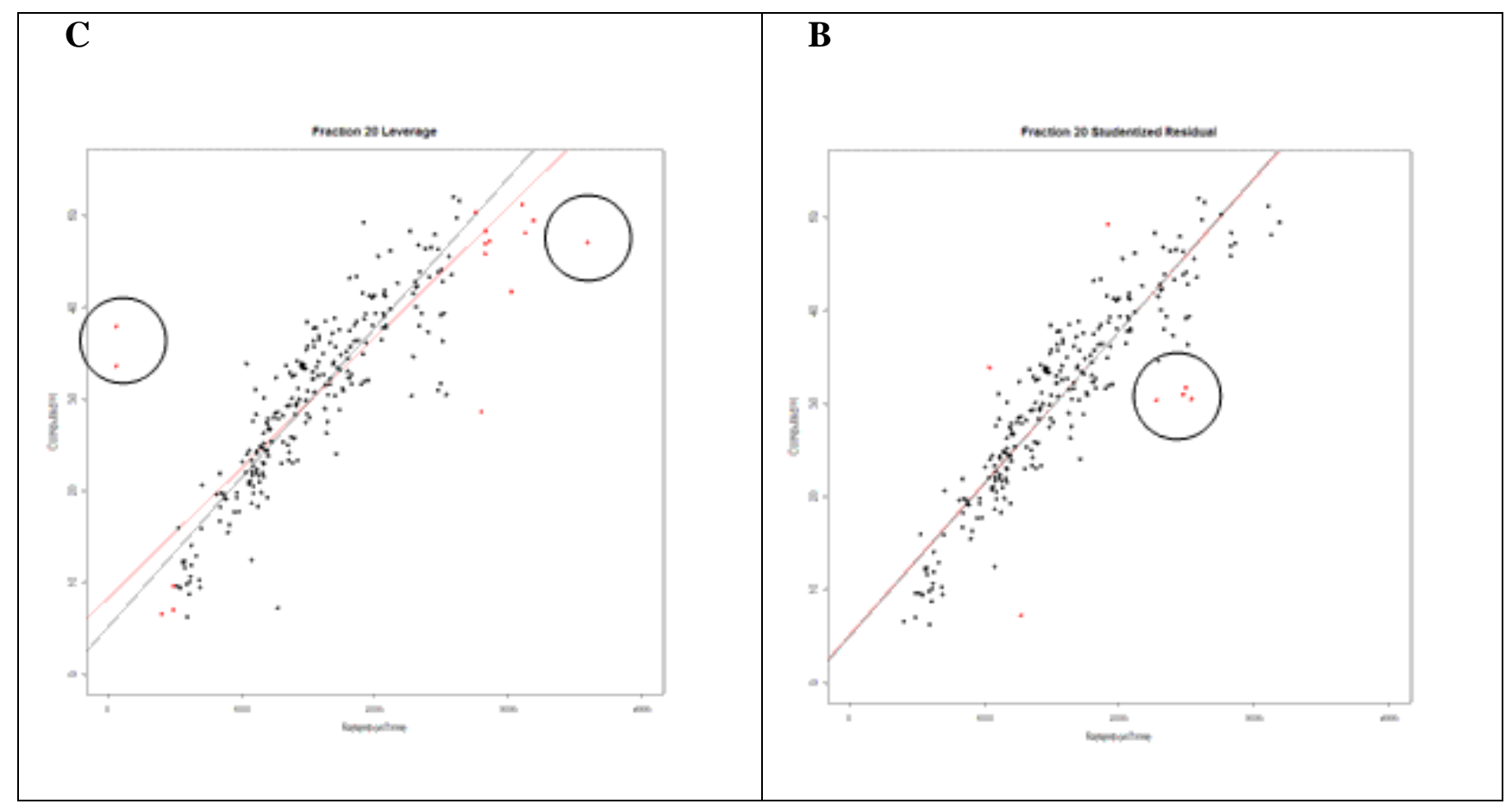

Figure 2. (A) Points with high leverage in red, other points in black. Linear regression based on all points: red line. Linear regression based on black points only: black line. (B) Points with studentized residual in red, other points in black. Linear regression based on all points: red line. Linear regression based on black points only: black line.

\section{AMT Database File Storage}

In order to preserve AMT databases, we have created a file format that encapsulates all necessary information about an AMT database. This file format conforms to an XML schema known as “amtXML”.

Some of the structure of amtXML is shown in Figure 3. At the root level is a single node representing the AMT database. Relatively little information is stored at this level - a count of the number of peptide entries, and the number of runs that contributed to the database. 
Within an AMT database, we define:

- The different amino acid modifications that are observed across all of the runs that make up the database. For instance, peptides may be observed with or without oxidized Methionine. These modifications may or may not have an effect on peptide retention time. They are ignored for the purposes of this manuscript, but this same data structure could be used to analyze the effects of modifications on retention time.

- The individual runs that make up the database. For each run, we store a number of attributes, including the linear mapping from RT to $\mathrm{H}$ that was used when creating that run's peptide observations, and when the run was added to the database. We also store a unique numeric ID for this run that can be referenced in individual peptide observations.

- All of the individual peptide entries that make up the bulk of the database. There is a median_observed_hydrophobicity attribute at this level that is used when matching to MS1 features.

Below the peptide entry level, more data about individual observations are kept, for use when recalculating median hydrophobicity after a change to the database. Each peptide entry is divided into one or more modification states (an indication of which amino acids, if any, contained modifications in this observation). Within each modification state, we have the individual observations from particular runs. Each observation contains an observed (normalized) hydrophobicity value, the PeptideProphet probability associated with the observation, and a reference to the run that it was observed in. Optionally, we may also keep track of the number of times that the peptide was observed in this run.

The XML schema document describing the amtXML file format (amtXml.xsd) is supplied with the msInspect source bundle, available at proteomics.fhcrc.org. 


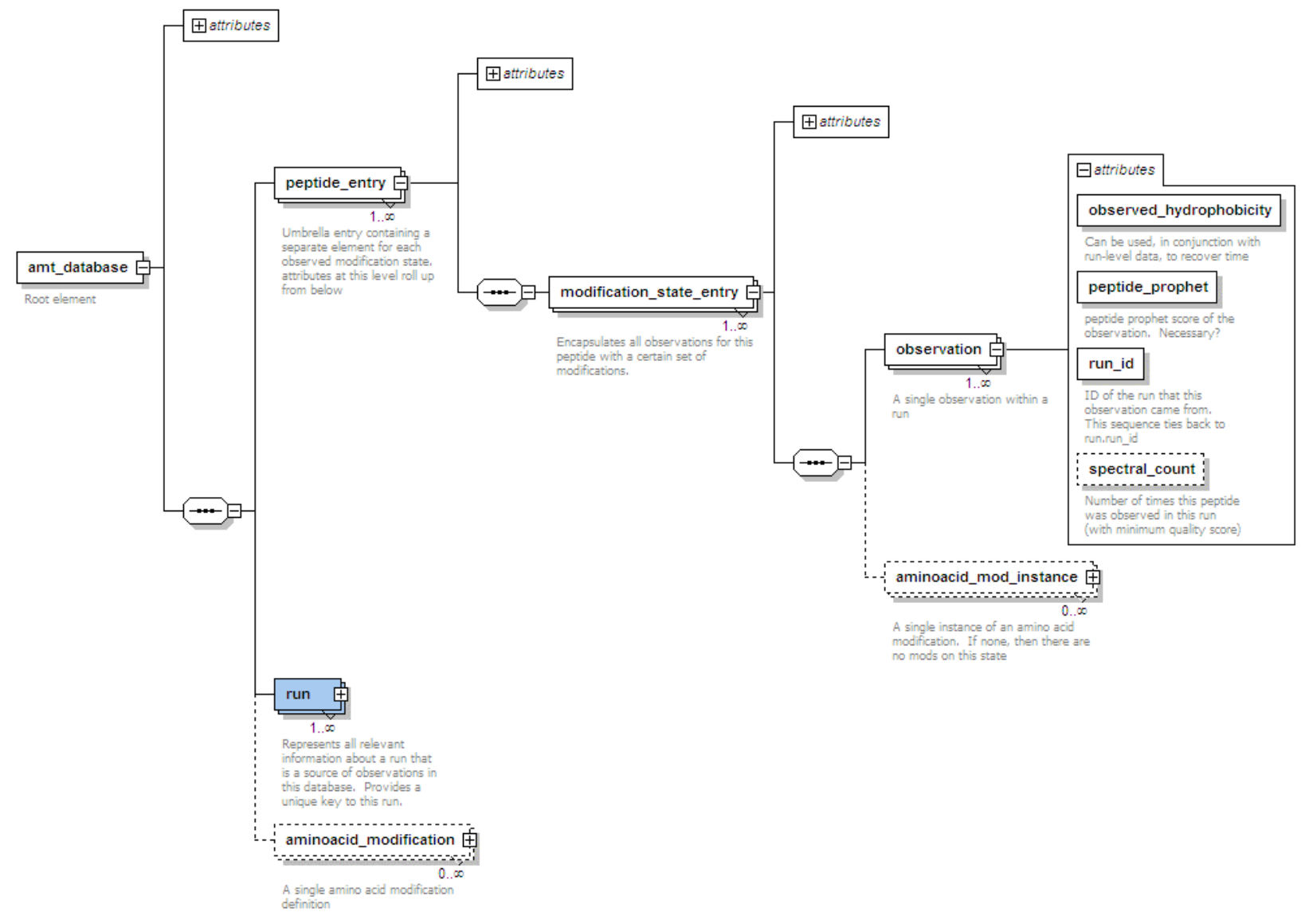

Figure 3. amtXML schema high-level summary.

\section{Quantile Regression}

In the AMT workflow, we use quantile regression to establish the relationship between Regression Time (RT) and Hydrophobicity $(\mathrm{H})$ in a given run. We begin with a set of $\{\mathrm{RT}, \mathrm{H}\}$ pairs for those LCMS peptides that match to peptides in the AMT database by tight mass-only matching. Neither ordinary least-square regression nor robust regression is effective in this situation, because the assumptions underlying least-squares estimation are questionable (see Figure 4). More specific, it is likely that the normal distribution assumption of the error terms was violated probably due to high proportion of miss matching pairs. 
The idea of quantile regression was first introduced by Koenker and Bassett (1978) and has been extended dramatically in the past two decades. It emerged as a powerful, robust alternative to leastsquares procedures for skewed data when the assumption of normality is violated. Quantile regression borrows the idea of minimizing the sum of absolute error (LAE) for median regression and generalizes the LAE estimator to quantiles, in which we minimize a sum of asymmetrically weighted absolute residuals by giving different weights to positive and negative residuals (Koenker 2001).

We modified the method of quantile regression to fit our situation. Instead of fixing quantile (parameter tau) in the regression, we developed an algorithm to search the optimal quantile so that the mode of residual from kernel density estimates is equal to zero. We estimate this optimized quantile regression line and predict the peptides' hydrophobicity using MS1 retention time. This method works very well when a high number of the peptides are correctly matched to the AMT database. The regression can be visually verified using plots as shown in Figure 2.

To use this method, both the $\mathbf{R}$ statistical language and the R package "quantreg" must be installed in the local computer. They are open-source software projects and can be freely downloaded from the CRAN repository: $\underline{\text { http://cran.r-project.org }}$ 

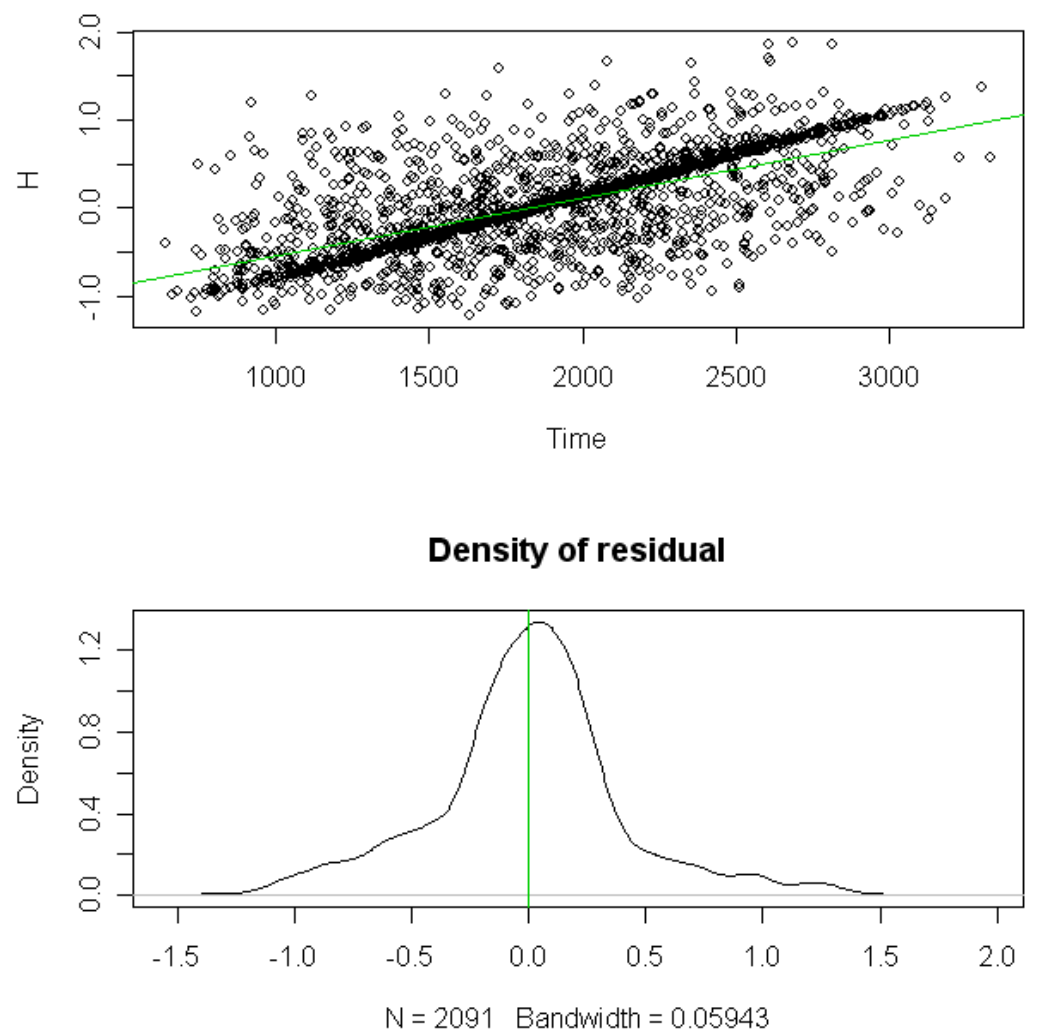

Figure 4: Using ordinary least square estimates. 

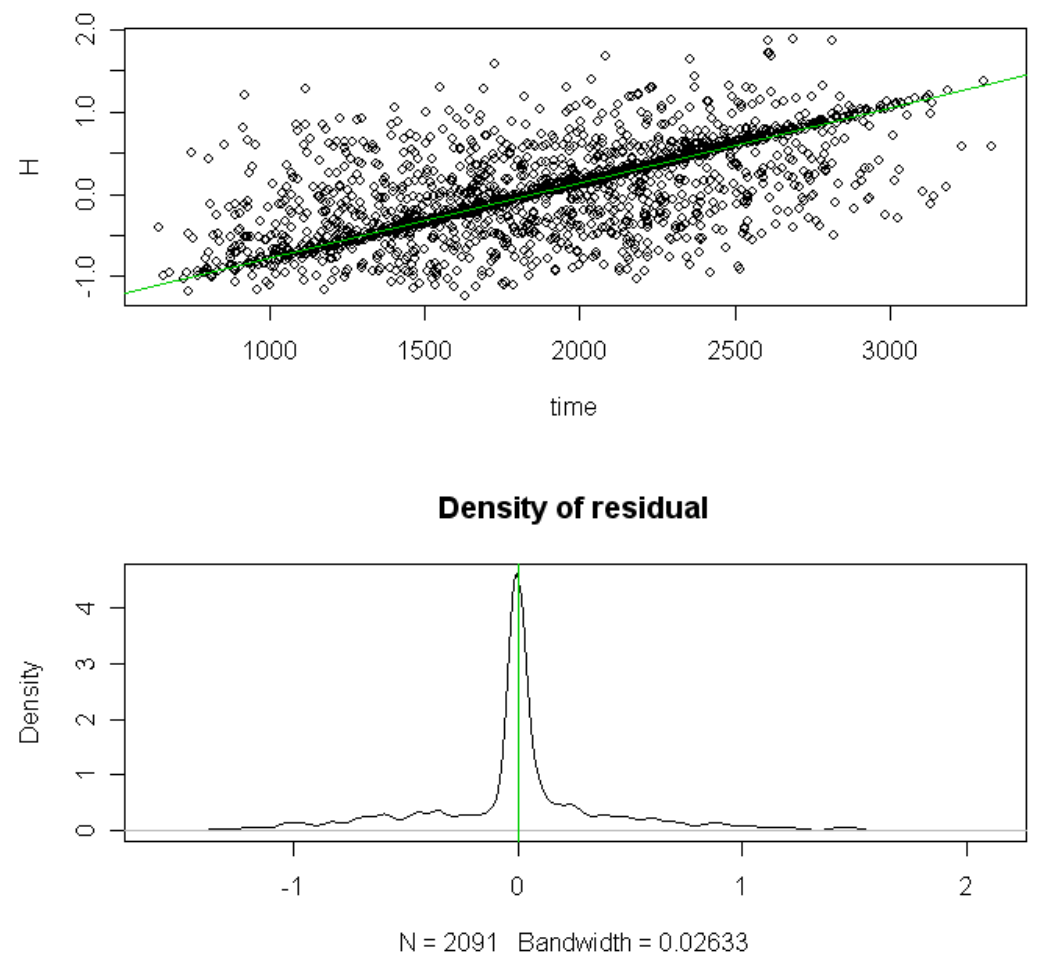

Figure 2: using quantile regression

\section{References}

(1) Koenker, Roger and Gilbert Bassett. (1978) "Regression auantiles.” Ecnometrica. 46:1, $33-50$

(2) Koenker, Roger and Hallock, Kevin F. (2001) "Quantile regression", Journal of economics 